\title{
Active control of strain in a composite plate using shape memory alloy actuators
}

\begin{abstract}
The present study aims to design an experimental test bench to analyze and control a smart structure system composed of a woven fiberglass laminate plate with shape memory alloy (SMA) actuators instilled on the surface. The aim of this design is to accurately augment the strain of the composite plate. Finite element analysis was employed to model the composite structure and determine the placement of the SMA actuators in order to produce the desired structural response efficiently with minimum power consumption. Due to the nonlinear behavior of the SMA actuator, it will be critical to incorporate a feedback control system that is able to accurately morph the structure by changing the strain of the composite structure. A Proportional-Integral-Derivative (PID) controller was designed to improve its tracking performance. Simulation on the control system showed that the PID controller produced acceptable response and managed to reduce steady state error for different types of input. The PID controller was then implemented in the experimental setup to control the smart composite plate. Results from the experiment illustrates that the smart structure system that has been designed per-formed effectively and the strain value of the composite structure can be controlled accurately.
\end{abstract}

Keywords: Smart structure system; Shape memory alloy; PID control; Composite 